\title{
Article \\ Self-Assembled Permanent Micro-Magnets in a Polymer-Based Microfluidic Device for Magnetic Cell Sorting
}

\author{
Lucie Descamps ${ }^{1}$ (D), Marie-Charlotte Audry ${ }^{1}$, Jordyn Howard ${ }^{1}$, Samir Mekkaoui ${ }^{1}$, Clément Albin ${ }^{2}$, \\ David Barthelemy ${ }^{3}$, Léa Payen ${ }^{3}$, Jessica Garcia ${ }^{3}$, Emmanuelle Laurenceau ${ }^{4} \mathbb{D}$, Damien Le Roy ${ }^{2, *}$ and \\ Anne-Laure Deman ${ }^{1, *}$
}

1 CNRS, INSA Lyon, Ecole Centrale de Lyon, CPE Lyon, INL, UMR5270, University Lyon, Université Claude Bernard Lyon 1, 69622 Villeurbanne, France; lucie.descamps@univ-lyon1.fr (L.D.); marie-charlotte.audry-deschamps@univ-lyon1.fr (M.-C.A.); jordyn.howard@etu.univ-lyon1.fr (J.H.); samir.mekkaoui@univ-lyon1.fr (S.M.)

2 CNRS, UMR5306 Institut Lumière Matière, University Lyon, Université Claude Bernard Lyon 1, 69100 Villeurbanne, France; clement.albin@univ-lyon1.fr

3 Laboratoire de Biochimie Et Biologie Moléculaire, Groupe Hospitalier Sud, Hospices Civils de Lyon, 69495 Pierre Bénite, France; david.barthelemy@chu-lyon.fr (D.B.); lea.payen-gay@chu-lyon.fr (L.P.); jessica.garcia@chu-lyon.fr (J.G.)

4 CNRS, INSA Lyon, CPE Lyon, CNRS, INL, UMR5270, University Lyon, Ecole Centrale de Lyon, Université Claude Bernard Lyon 1, 69130 Ecully, France; emmanuelle.laurenceau@ec-lyon.fr

* Correspondence: damien.le-roy@univ-lyon1.fr (D.L.R.); anne-laure.deman-him@univ-lyon1.fr (A.-L.D.)

check for updates

Citation: Descamps, L.; Audry, M.-C.; Howard, J.; Mekkaoui, S.; Albin, C.; Barthelemy, D.; Payen, L.; Garcia, J.; Laurenceau, E.; Le Roy, D.; et al. Self-Assembled Permanent Micro-Magnets in a Polymer-Based Microfluidic Device for Magnetic Cell Sorting. Cells 2021, 10, 1734. https:// doi.org/10.3390/cells10071734

Academic Editors: Vitalii Zablotskii and Xin Zhang

Received: 26 May 2021

Accepted: 5 July 2021

Published: 9 July 2021

Publisher's Note: MDPI stays neutral with regard to jurisdictional claims in published maps and institutional affiliations.

Copyright: (c) 2021 by the authors. Licensee MDPI, Basel, Switzerland. This article is an open access article distributed under the terms and conditions of the Creative Commons Attribution (CC BY) license (https:/ / creativecommons.org/licenses/by/ $4.0 /)$

\begin{abstract}
Magnetophoresis-based microfluidic devices offer simple and reliable manipulation of micro-scale objects and provide a large panel of applications, from selective trapping to highthroughput sorting. However, the fabrication and integration of micro-scale magnets in microsystems involve complex and expensive processes. Here we report on an inexpensive and easy-to-handle fabrication process of micrometer-scale permanent magnets, based on the self-organization of $\mathrm{NdFeB}$ particles in a polymer matrix (polydimethylsiloxane, PDMS). A study of the inner structure by X-ray tomography revealed a chain-like organization of the particles leading to an array of hard magnetic microstructures with a mean diameter of $4 \mu \mathrm{m}$. The magnetic performance of the self-assembled micro-magnets was first estimated by COMSOL simulations. The micro-magnets were then integrated into a microfluidic device where they act as micro-traps. The magnetic forces exerted by the micro-magnets on superparamagnetic beads were measured by colloidal probe atomic force microscopy (AFM) and in operando in the microfluidic system. Forces as high as several nanonewtons were reached. Adding an external millimeter-sized magnet allowed target magnetization and the interaction range to be increased. Then, the integrated micro-magnets were used to study the magnetophoretic trapping efficiency of magnetic beads, providing efficiencies of $100 \%$ at $0.5 \mathrm{~mL} / \mathrm{h}$ and $75 \%$ at $1 \mathrm{~mL} / \mathrm{h}$. Finally, the micro-magnets were implemented for cell sorting by performing white blood cell depletion.
\end{abstract}

Keywords: magnetophoresis; micro-magnets; polymer composite; microfluidic devices; particle separation

\section{Introduction}

Microfluidic magnetophoresis has been demonstrated as an efficient way to trap and separate biological entities and is now integrated in lab-on-chip systems for various biomedical applications, including clinical diagnosis [1,2]. By general principle, biological entities-whether proteins [3-5], DNA [6-8], or cells [9-11] —are magnetically labeled with nano- or microparticles and are dragged towards local magnetic field maxima, generated by magnetic flux sources, with a high selectivity [12].

For a given target magnetic particle, the highest attainable force is limited to the field gradient of the magnetic field source. It is therefore of primary interest to downsize the 
magnetic field source as it scales up the magnetic field gradients. With micrometer-sized magnets, local magnetic field gradients as high as $10^{6} \mathrm{~T} / \mathrm{m}$ have been reported in multipole structures [13].

A high density of such micro-traps is needed to efficiently sort entities in fluidic samples. To this end, micropatterning films produced through lithography [14], chemical etching [15,16], or by deposition on topographically structured substrates [17] have been reported. Regardless of the employed microfabrication technique, this film-based approach qualifies as a "top-down" method, offers mastered shapes, and remarkable reproducibility. However, the heterogeneous integration of metals in polymer-based devices raises cohesiveness issues that can be overcome by transferring the synthesized metallic micropatterns in a polymer host matrix [17]. A drastically different approach using a "bottom-up" principle consists of powder compaction and bonding. The host polymer matrix facilitates the integration in polymer-based devices, however, the heterogeneous particle size and morphology lead to less control over the shape and reproducibility of the array of micro-traps [18]. Among the developed approaches, microstructure engineering of powder-polymer mixtures has recently emerged as a noteworthy breakthrough. In principle, the magnetic powder spatial distribution is driven by a magnetic field template during the reticulation of the polymer matrix. It offers a process to reach complementary morphologies, with respect to standard film-based micropatterning processes, without requiring expensive and complex experimental set-up [19].

Here we present a simple fabrication process based on $\mathrm{NdFeB}$ particles self-ordering in a polymer matrix, polydimethylsiloxane (PDMS), which is widely used in microfluidic systems. The magnetic forces generated by the NdFeB@PDMS microstructure were simulated in COMSOL and measured with both colloidal probe atomic force microscopy (AFM) and in operando through hydrodynamic determination. We found that magnetic forces up to few $\mathrm{nN}$ are reached at contact, and the interaction distance and the magnetization of the target could be increased by adding an external millimeter-sized permanent magnet. This dual-scale permanent magnet was integrated into a microfluidic channel to first measure magnetic bead trapping efficiencies, and then to perform magnetically labeled white blood cell (WBC) trapping.

\section{Materials and Methods}

\subsection{Materials}

A Sylgard Silicone Elastomer (PDMS, Polydimethylsiloxane) was purchased from Samaro (Beynost, France) and consists of two components: a base and a curing agent (10:1 mixing ratio). NdFeB micro-particles are irregularly shaped crushed melt-spun ribbon (MQFP-B, 0.5-7 $\mu \mathrm{m}$ size) provided by Magnequench International, Inc. (Singapore). An SEM image of the NdFeB micro-particles can be found in Figure S1 (see Supporting Information). The magnetic properties of the particles were as follows: remanence, $\mu_{0} \mathrm{M}_{\mathrm{r}}=0.9 \mathrm{~T}$ ( $\mu_{0}$ being the permeability of free space); and coercivity field, $\mathrm{H}_{\mathrm{c}}=740 \mathrm{kA} / \mathrm{m}$. A millimeter-sized permanent magnet $\left(25 \times 8 \times 2 \mathrm{~mm}^{3}\right.$, remanent magnetization $\mu_{0} \mathrm{M}_{\mathrm{R}} \sim 1.4 \mathrm{~T}$, Supermagnete, Gottmadingen, Germany) was also used to study magnetic performance enhancement. Superparamagnetic microbeads (SPMBs) (average diameter: $12 \mu \mathrm{m}$, density: $1.1 \mathrm{~g} / \mathrm{cm}^{3}$, magnetization: $0.66 \mathrm{kA} / \mathrm{m}$, material: magnetite nano-inclusions in a polystyrene matrix, 1 vol $\% \mathrm{Fe}_{3} \mathrm{O}_{4}$ ) were purchased from Kisker Biotech $\mathrm{GmbH} \&$ Co. (Steinfurt, Germany) to perform colloidal probe AFM measurements and fluidic experiments.

\subsection{Device Fabrication}

\subsubsection{Composite Membrane Preparation}

Micro-magnet fabrication is based on the composite approach which consists of mixing a hard magnetic powder with a polymer material. NdFeB@PDMS composite membranes were fabricated with concentrations of $2 \mathrm{wt} \% \mathrm{NdFeB}$. A flowchart describing the fabrication steps of the micromagnets is reported in Figure 1. Briefly, the mixture containing NdFeB micro-particles and uncured PDMS was poured into a 100- $\mu$ m-thick Kapton mold stuck to 
a silanized glass slide [20]. The composite was then cured at $70{ }^{\circ} \mathrm{C}$ for $2 \mathrm{~h}$ in a magnetic field of $300 \mathrm{mT}$ supplied by a bulk $\mathrm{NdFeB}$ magnet $\left(60 \times 30 \times 15 \mathrm{~mm}^{3}\right.$, magnetization along the shortest dimension) to allow PDMS cross-linking and NdFeB particle self-assembly. After Kapton mold removal, the thickness of the composite membrane was increased to $1 \mathrm{~mm}$ by pouring liquid PDMS and curing the ensemble at $70{ }^{\circ} \mathrm{C}$ for $2 \mathrm{~h}$. Finally, the NdFeB@PDMS membrane was peeled off the glass substrate and $\mathrm{NdFeB}$ microstructures were magnetized using a homemade magnetizing system (two magnets of dimensions $50.8 \times 50.8 \times 25.4 \mathrm{~mm}^{3}$, spaced $2.5 \mathrm{~mm}$ from each other) that produced a field of $1 \mathrm{~T}$.

1)

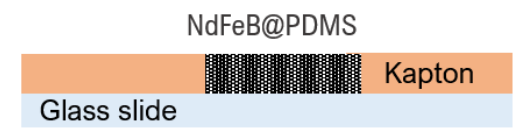

2)

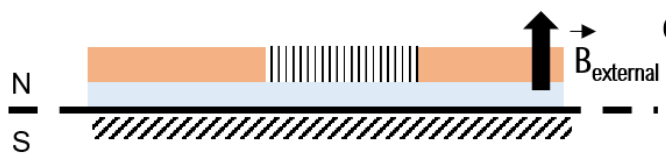

3)

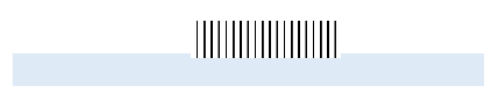

4)

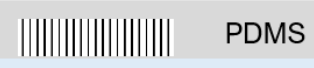

5)

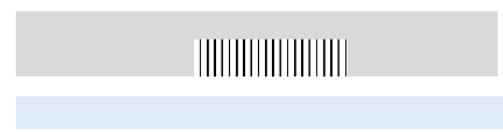

6)

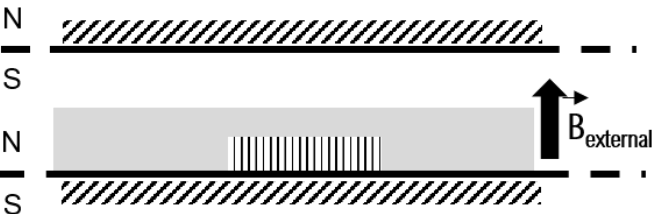

7)

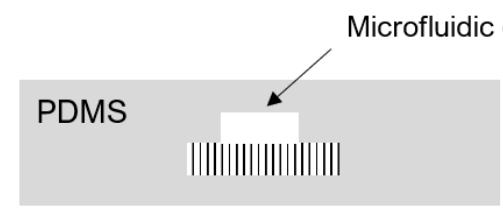

Figure 1. Fabrication steps of the microdevice: (1) the composite is molded in a Kapton film bonded to a silanized glass slide substrate; (2) the composite is then placed in a $300 \mathrm{mT}$ magnetic field for NdFeB particle self-organization in chains at $60{ }^{\circ} \mathrm{C}$ for $2 \mathrm{~h} ;(3,4)$ the Kapton mold is then removed and pure PDMS is poured; (5) after curing at $70{ }^{\circ} \mathrm{C}$ for $2 \mathrm{~h}$, the composite membrane is peeled off and (6) magnetized under a magnetic field of $1 \mathrm{~T}$. Finally, (7) the composite membrane is bonded to a channel molded in PDMS by $\mathrm{O}_{2}$ surface plasma activation.

\subsubsection{Whole Device Assembly}

The micro-magnet array was integrated into a microfluidic system by sealing the composite membrane with PDMS microfluidic channels using $\mathrm{O}_{2}$ plasma bonding. The channel mold $\left(40 \times 0.5 \times 0.1 \mathrm{~mm}^{3}\right)$ was obtained by soft-lithography using a $50-\mu \mathrm{m}$ dry photoresist (Eternal Materials Co., Etertec ${ }^{\circledR}$, Kaohsiung City, Taiwan).

\subsection{Characterization}

\subsubsection{Structural Characterization}

The inner structure of the composite membrane was characterized by X-ray tomography, using the EasyTom Micro and Nano CT Tomography System (RX Solutions). The X-ray source was a LaB6 cathode with a diamond window leading to higher flux $(20 \mu \mathrm{A})$. Its focal spot measured $0.25 \mu \mathrm{m}$ and a tension of $90 \mathrm{kV}$ was applied. Scans were acquired by a CCD detector, whose matrix measured $2000 \times 1312$ pixels, with a voxel resolution of $0.3 \mu^{3}$. 3D images were reconstructed from projections at 1400 different angular positions. Final images of $1700 \times 1700 \times 400$ voxel, i.e., $510 \times 510 \times 120 \mu^{3}$, were obtained and processed with ImageJ to characterize $\mathrm{NdFeB}$ particles' spatial organization in the volume of the composite membrane. Conventional optical microscopy characterizations using an Olympus BX51M microscope coupled to a camera (Moticam2000, Motic ${ }^{\circledR}$, Wetzlar, Germany) were also carried out with ImageJ to study the in-plane organization of the $\mathrm{NdFeB}$ agglomerates. 


\subsubsection{Magnetic Characterization}

Magnetization and demagnetization processes were measured in a SQUID magnetometer (Quantum Design MPMS XL). The characterized sample was a 100- $\mu \mathrm{m}$-thick, 4-mm-sided square piece of NdFeB@PDMS membrane, bonded to a 1-mm-thick PDMS support. The columnar NdFeB agglomerates pointed along the membrane's thickness. The sample was fixed in a straw perpendicularly using Kapton tape. Therefore, the applied magnetic field in the SQUID was directed along the long axis of the NdFeB chain agglomerates, similar to the set-up of the operational device.

\subsubsection{Magnetic Force Measurements}

Magnetic force measurements were carried out on the AFM MFP-3D (Asylum Research, Oxford Instrument, Goleta, CA, USA) using a 15- $\mu$ m superparamagnetic colloidal probe (SPMB) glued to a silicon nitride cantilever (PNP-TR-TL, stiffness measured with thermal noise method: $43 \mathrm{pN} / \mathrm{nm}$, NanoAndMore, Paris, France). The acting force between the colloidal probe and the sample was recorded as the probe approached and withdrew from the sample surface. In particular, the sample was scanned in two-pass mode, as follows: the first scan was performed at contact, to localize the micro-magnets, and the second one at a distance of a few hundred nanometers, to record the cantilever's deflection above the sample surface. In this way, the maximum force generated by the localized micro-magnet can be measured, and the approach/retract curve is performed at the exact micro-magnet position (see Figure S2) at a $1 \mu \mathrm{m} / \mathrm{s}$ constant velocity. Measurements were performed in a $10 \mathrm{mM} \mathrm{NaCl}$ solution to screen electro-static forces. A total of 40 micro-magnets were characterized using this method.

\subsection{Bead Injection}

SPMBs were suspended in a filtered phosphate buffer saline (PBS, Sigma-Aldrich, Saint-Louis, MO, USA) solution with $2 \%$ pluronic F-108 (Sigma-Aldrich, Saint-Louis, $\mathrm{MO}, \mathrm{USA})$ at a concentration of $50 \mathrm{SPMBs} / \mu \mathrm{L}$ and injected into the microchannel using a pressure-driven flow controller (Flow-EZ ${ }^{\mathrm{TM}}$, Fluigent, Le Kremlin-Bicêtre, France). A picture of the experimental set-up is provided in Supporting Information (Figure S3).

\subsection{Cell Sample Preparation}

WBCs were collected from a whole blood sample after red blood cell lysis and were magnetically labeled with superparamagnetic nanoparticles (Ademtech SA, Pessac, France, MasterBeads Carboxylic Acid 0215). The nanoparticles were $500 \mathrm{~nm}$ in diameter and composed of a magnetic core (approximately $70 \%$ iron oxide) encapsulated by a hydrophilic polymer shell with carboxyl groups on its surface. They were functionalized with both anti-CD45 and anti-CD15 antibodies (purchased from R \& D Systems, Minneapolis, MO, USA) to enhance WBC magnetic labeling. Finally, WBCs were suspended in $500 \mu \mathrm{L}$ of EDTA ( $2 \mathrm{mM}$, PBS-BSA $2 \%$ ) at a concentration of $6.10^{5} \mathrm{WBCs} / \mathrm{mL}$. PC-3 cancer cells were spiked into the white blood cell sample, at a concentration of $4.10^{4} \mathrm{PC}-3 / \mathrm{mL}$, to study the specificity of the trapping. WBCs were stained with Hoechst 33342 (Ready Flow Reagent ${ }^{\mathrm{TM}}$, ThermoFisher Scientific, Waltham, MA, USA) and PC-3 cells were tracked with a green dye (CellTracker ${ }^{\mathrm{TM}}$ Green CMFDA Dye, ThermoFisher Scientific, Waltham, MA, USA).

\section{Results and Discussion}

\subsection{Micro-Magnet Microstructure and Magnetic Performances}

The reconstructed 3D profile of the NdFeB@PDMS membrane from X-ray tomography observation is shown in Figure 2a, revealing a predominantly chain-like organization of NdFeB micro-particles in the PDMS matrix. This chain organization is particularly visible in Figure $2 \mathrm{~b}$, where a 100- $\mu \mathrm{m}$-thick slice of the composite is presented in the $(y, z)$ plane. During the PDMS cross-linking step, in which an external magnetic field is applied, the NdFeB particles, seen as magnetic dipoles, are driven by dipole-dipole interactions [21]. A nearly homogeneous applied field favors a uniaxial stacking of the 
particles along the field direction and a repulsion in the perpendicular directions [22]. The X-ray tomography images of the self-assembled microstructures were characterized using ImageJ. The average length of the microstructures is in the order of $75 \mu \mathrm{m}$ and the interparticle distance can reach 0.4 to $4 \mu \mathrm{m}$ for some chains, knowing that the resolution of X-ray tomography is $0.3 \mu \mathrm{m}$. Although the chains present a certain heterogeneity, the composite approach allows the integration of micro-magnet arrays in PDMS without complex and costly technological steps. A top view of the micro-magnets is presented in Figure $2 \mathrm{c}$ using brightfield microscopy. Their diameter ranges between 0.6 and $18 \mu \mathrm{m}$, of which $98 \%$ are smaller than $10 \mu \mathrm{m}$. The nearest neighbor distance (nnd) distribution is shown in Figure 2d, with an average distance of $16 \mu \mathrm{m}$. In general, the Poisson law can describe independent events and has no adjustable parameters. Applied to a given number of particles on a known surface, it allows the description of a random distribution. Here, it fails to describe the experimental nnd distribution as obtained from brightfield microscopy observation. It is better described by a normal distribution, demonstrating that the self-organization of the micro-magnets is not random. The total density of the magnetic microstructures reached $1250 \pm 130 / \mathrm{mm}^{2}$.

a)

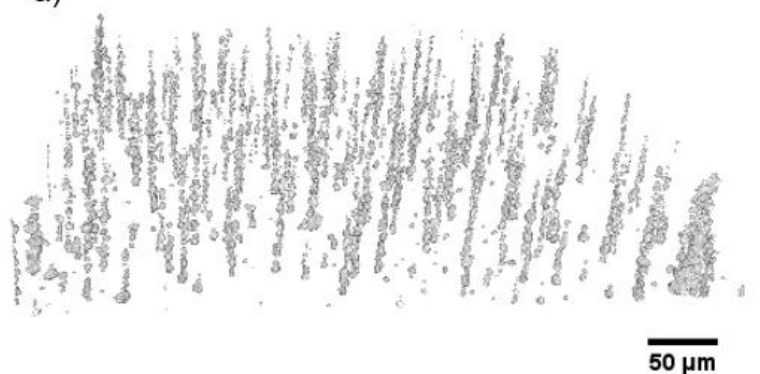

c)

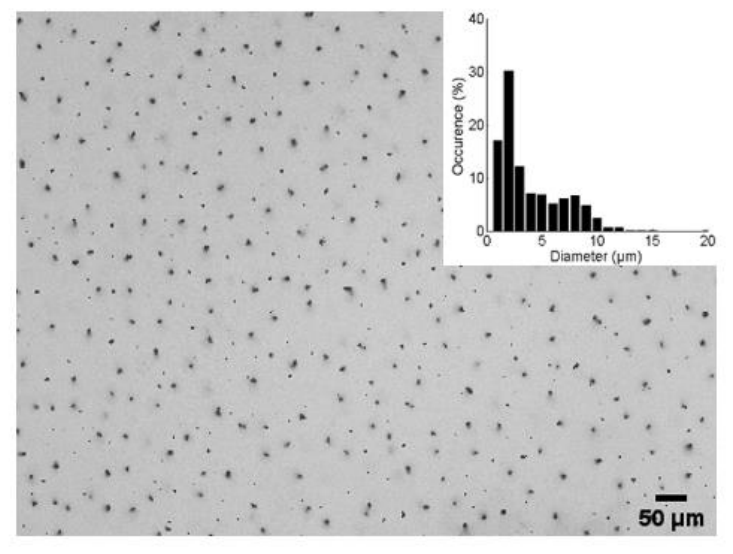

b)

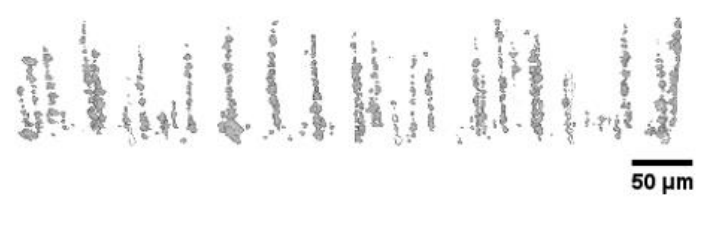

d)

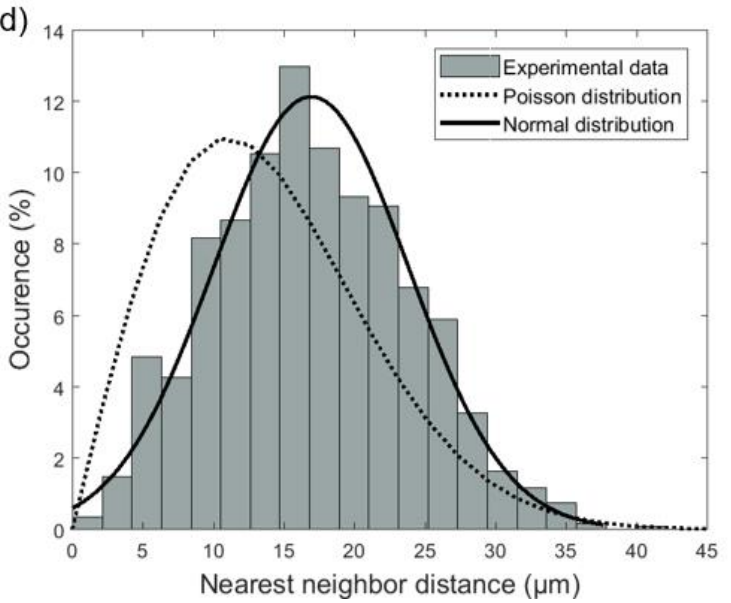

Figure 2. (a) $2 \% \mathrm{NdFeB}$ particle organization in PDMS membrane from X-ray tomography reconstruction of a $510 \times 510 \times 120 \mu \mathrm{m}^{3}$ volume. (b) Section view of the chain-like organization of the $2 \% \mathrm{NdFeB}$ particles obtained from X-ray tomography reconstruction of a $100 \times 510 \times 120 \mu \mathrm{m}^{3}$ volume. (c) Microscope image of the micro-magnet array (top view), and (inset) the micro-magnet diameter distribution. (d) Nearest neighbor distance distribution. The experimental distance, measured from brightfield microscopy observation $\left(0.8 \mathrm{~mm}^{2}\right.$ images), followed a normal distribution and not a Poisson distribution (random distribution of $\mathrm{N}=1225$ particles in a $0.8 \mathrm{~mm}^{2}$ frame).

Prior to its implementation in a microfluidic device, the magnetization of the NdFeB@PDMS composite membrane was measured in a SQUID magnetometer at room temperature. The out-of-plane magnetization loop is shown in Figure 3a, specifically when the field is applied along the agglomerates' long axis. In order to determine the operating magnetization state, first magnetization curves were measured with successive returns to the remanent state. The first point, $\mathrm{M}_{\mathrm{RI}}$ (initial remanent magnetization), indicates the 
remanent magnetization after the field reticulation process. $\mathrm{M}_{\mathrm{RF}}$, corresponding to the full remanent magnetization, was $0.75 \mathrm{M}_{\mathrm{S}}\left(\mu_{0} \mathrm{M}_{\mathrm{RF}}=0.84 \mathrm{~T}\right)$, and was reached with a field larger than $2.5 \mathrm{~T}$. $\mathrm{M}_{\mathrm{RO}}$ (operating remanent magnetization) corresponds to the magnetization after magnetizing the membrane in a $1 \mathrm{~T}$ field, which is equivalent to the field used in the magnetizing process of the operating device. $\mathrm{M}_{\mathrm{RO}}$ reached $0.48 \mathrm{M}_{\mathrm{S}}$, and we can estimate the remanent magnetic field of the micro-traps, $\mu_{0} \mathrm{M}_{\mathrm{RO}}$, to be $0.54 \mathrm{~T}$.

a)

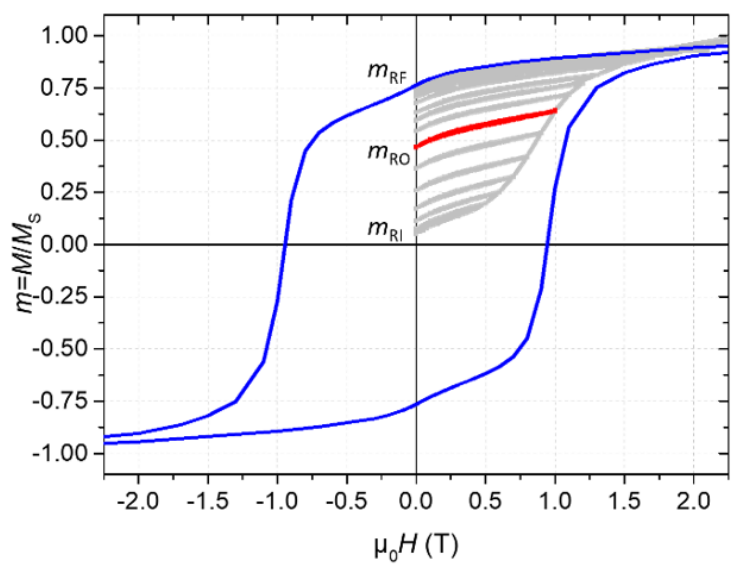

b)

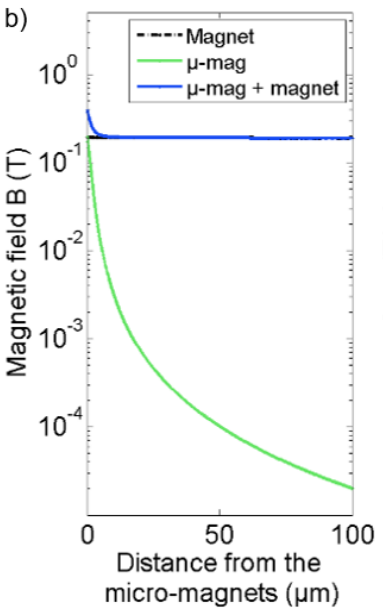

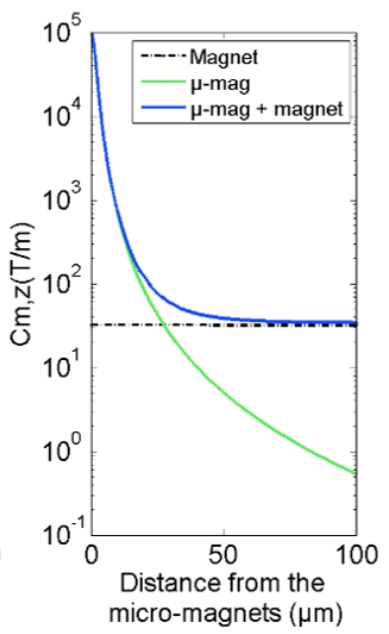

Figure 3. (a) Magnetization of the NdFeB@PDMS membrane, measured out-of-plane. The light grey curve is the first magnetization curve with successive measurements of remanent magnetization. The thick red curve highlights the remanent magnetization at $1 \mathrm{~T}$. The blue curve is the full magnetization loop. (b) COMSOL simulations of the magnetic field and magnetic field gradient (defined by $\mathrm{Cm}, \mathrm{z}$ ) generated by the micro-magnets ( $\mu$-mag) as a function of the distance from the composite surface, with or without an external magnet below the composite surface.

The magnetic properties of the organized NdFeB micro-magnets in the PDMS matrix were modeled using a finite element approach (COMSOL, AC/DC module, 2D axisymmetric model). Based on X-ray tomography observations, an individual chain-like structure was modeled as 28 cylindrical NdFeB particles of diameter $4 \mu \mathrm{m}$ and height $1.5 \mu \mathrm{m}$, each spaced apart by $1.2 \mu \mathrm{m}$ (total chain length of $75 \mu \mathrm{m}$ ). From SQUID measurements, the remanent field of the particles was set at $0.54 \mathrm{~T}$. The magnetic field and magnetic field gradient were calculated as a function of the distance from the composite surface (Figure 3b). The magnetic field gradient generated by the magnetic structure, along the $\mathrm{z}$-direction, is pictured by a magnetic coefficient $(\mathrm{Cm}, \mathrm{z})$, defined as follows:

$$
\mathrm{Cm}, \mathrm{z}=\frac{1}{\sqrt{\mathrm{B}_{\mathrm{r}}^{2}+\mathrm{B}_{\mathrm{z}}^{2}}}\left(\mathrm{~B}_{\mathrm{r}} \frac{\partial \mathrm{B}_{\mathrm{z}}}{\partial \mathrm{r}}+\mathrm{B}_{\mathrm{z}} \frac{\partial \mathrm{B}_{\mathrm{z}}}{\partial \mathrm{z}}\right)
$$

The expression of $\mathrm{Cm}, \mathrm{z}$ was obtained from the development of the expression of the magnetic force (see the Supporting Information).

The microstructure generates a magnetic field of $200 \mathrm{mT}$ and a magnetic field gradient of $10^{5} \mathrm{~T} / \mathrm{m}$ at its surface, which are adequate with regard to numerical values found in the literature [23-25]. In turn, the magnetic field and the magnetic field gradient then decreased with the distance to the microstructure, down to $0.1 \mathrm{mT}$ and $5 \mathrm{~T} / \mathrm{m}$, respectively, at a distance of $50 \mu \mathrm{m}$ (Figure 3b). Magnetic objects initially flowing at the top of the channel may not be subjected to the relatively short interaction range of the micro-magnets, limiting the trapping efficiency in a 100- $\mu$ m-high channel. Therefore, we quantified the effect of adding an external millimeter-sized permanent magnet under the composite membrane (at a distance of $1 \mathrm{~mm}$ from the micro-magnets). Thus, as compared with the configuration with micro-magnets only, the combined use of the micro-magnets and milli-magnets doubled the magnetic field value in contact and increased the minimum value inside the channel from $0.02 \mathrm{mT}$ to $200 \mathrm{mT}$. The higher field value ensures a higher 
magnetic moment held by the target objects. Furthermore, the magnetic field gradient increased from $5 \mathrm{~T} / \mathrm{m}$ to $40 \mathrm{~T} / \mathrm{m}$ at a distance of $50 \mu \mathrm{m}$ (Figure $3 \mathrm{~b}$ ), therefore increasing the interaction distance [12].

\subsection{Generated Magnetic Forces}

We used colloidal probe AFM to measure the magnetic force produced by individual micro-traps. The SPMB, attached to the tipless AFM cantilever, was approached, then retracted from the composite's surface. The attractive force acting on the probe, measured by the cantilever deflection, is linked to the magnetic force. Contact forces ranging from 0.5 to $2 \mathrm{nN}$ were measured (Figure $4 \mathrm{a}$ ), and these values are in agreement with the data found in the literature for microscale soft magnetic sources [26-28] and hard magnetic structures [29]. The mapping of the magnetic attraction, performed at a distance of $500 \mathrm{~nm}$ from the surface, highlights that the maximum force is localized above the micro-magnet (Figure 4a, inset). Regarding the influence of an external permanent magnet, the magnetic force range was increased to $4 \mathrm{nN}$. Indeed, the magnetic force acting on the superparamagnetic bead of magnetization $\mathrm{M}_{\mathrm{b}}$ and volume $\mathrm{V}_{\mathrm{b}}$ is given by $\overrightarrow{\mathrm{F}_{\mathrm{mag}}}=\mu_{0} \mathrm{~V}_{\mathrm{b}}(\overrightarrow{\mathrm{M}} \mathrm{b} \cdot \vec{\nabla}) \overrightarrow{\mathrm{H}}$, where $\mathrm{H}$ is the applied magnetic field. The magnetization of SPMB (see Figure S4), when positioned at the composite's surface (i.e., in a field of $200 \mathrm{mT}$ ), is not fully saturated, and so the higher magnetic field value with the addition of the milli-magnet drove it up by $10 \%$ (from 470 to $510 \mathrm{~A} / \mathrm{m}$, Figure S4). Similarly, the magnetization of the micro-traps was slightly raised by $5 \%$ (from $490 \mathrm{kA} / \mathrm{m}$ to $515 \mathrm{kA} / \mathrm{m}$, Figure S5).
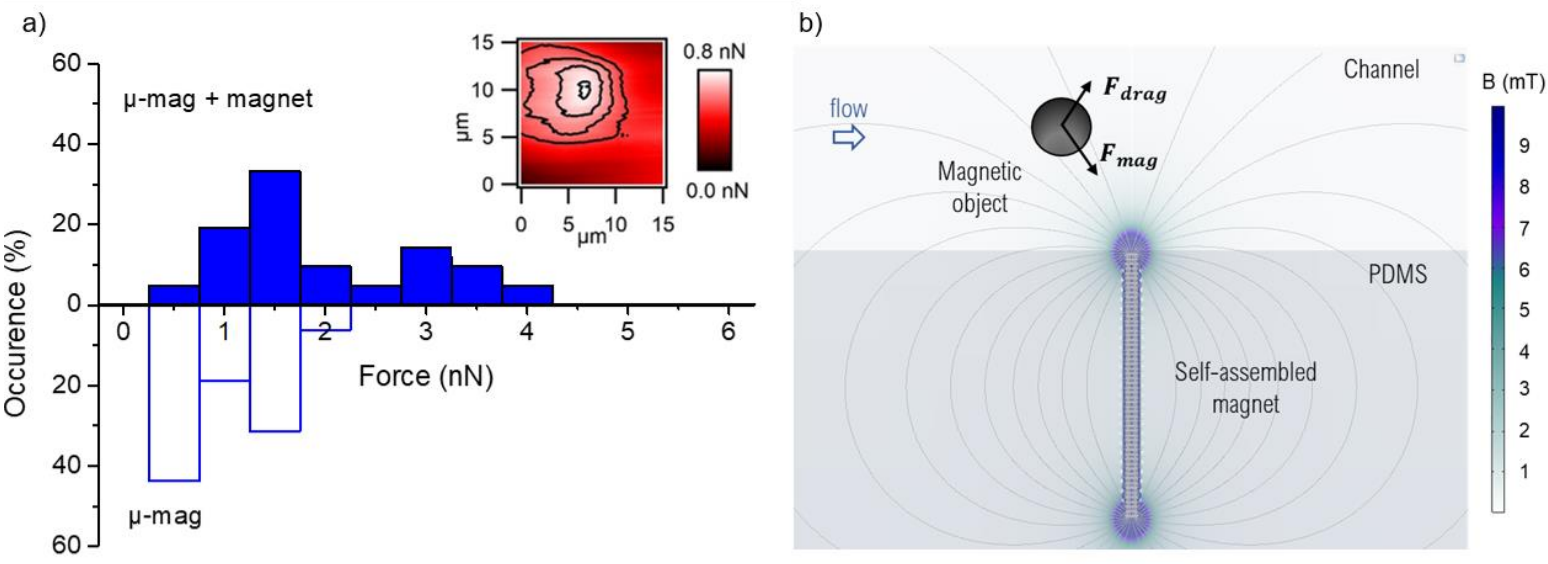

Figure 4. (a) Distribution of the magnetic force produced by the micro-magnets ( $\mu$-mag) from colloidal probe AFM measurements, with or without the external milli-magnet. The inset shows an example of a second scan in two-pass mode, at $500 \mathrm{~nm}$ above a micro-magnet (no external magnet), in order to localize the force of maximum intensity. (b) Schematic representation of the forces acting on a magnetic target flowing in a microfluidic device with magnetic structures located at the bottom of the channel.

Considering the low SPMB concentration, we used the single-particle-transport model to analyze the involved forces [30]. Moreover, given the low magnetic moment of the micro-beads, we neglected the dipole interactions and the contribution of their residual magnetization. Due to the micrometric size of the SPMBs, mass diffusion and magnetic diffusion based on Brownian motion can be ignored [31]. Finally, the buoyancy and gravity exerted on SPMBs are negligible, as compared with the magnetic and drag forces [2]. Thus, in the magnetic microfluidic system, the two main forces of importance to evaluate trapping efficiency are the magnetic force and Stokes' drag force (Figure $4 \mathrm{~b}$ ). Reliable trapping is obtained when the magnetic force value exceeds that of the drag force. We estimated the magnetic force generated by the micro-magnets, in operando, through the hydrodynamic determination of the holding magnetic force [19,32]. SPMBs were injected at an equivalent flow rate of $2 \mathrm{~mL} / \mathrm{h}$ and trapped on micro-magnets. Then, we injected PBS and gradually increased the flow rate and identified, for each trapped bead, the 
flow velocity, and thus the shear stress, at which it was untrapped. Average forces of $1.3 \pm 0.5 \mathrm{nN}$ were measured on more than a thousand beads, which is in good agreement with the forces measured by colloidal probe AFM. We also observed that traps located at the beginning of the trapping area generated greater forces than those located in the middle of the trapping zone. In particular, those located in the first $50 \mu \mathrm{m}$ of the trapping zone generated forces on average 1.8 times greater than those produced overall in the trapping zone, as shown in Figure 5a. This observation is in good agreement with the finite element simulation calculations of the field gradients generated by a network of chains (COMSOL, $\mathrm{AC} / \mathrm{DC}$ module, 2D model). We first determined the number of chains from which the network can be considered as an infinite number of chains, ensured by a variation of $\mathrm{Cm}$ intensity lower than 1\%. This threshold was found to be nine chains (see Figure S6). Then, the magnetic field gradient generated above a network of nine chains was compared with the one produced by a single chain (Figure 5b). 2D calculations show that a chain included in a network produces a $\mathrm{Cm}$ that is $34 \%$ lower than an isolated chain. As for the broken symmetry at the edge of the network, its effect on $\mathrm{Cm}$ is significant over a distance of about $50 \mu \mathrm{m}$ from the edge and the $\mathrm{Cm}$ produced by a chain on the side is $15 \%$ greater than that of a chain included in the network. These observations show the demagnetizing interaction between neighboring chains which tends to decrease the generated magnetic force. This demagnetizing effect inevitably occurs in any dense micro-array of magnetic structures. In these types of applications, there is always a tradeoff between the density of the micromagnets and their individual trapping efficacy.

a)

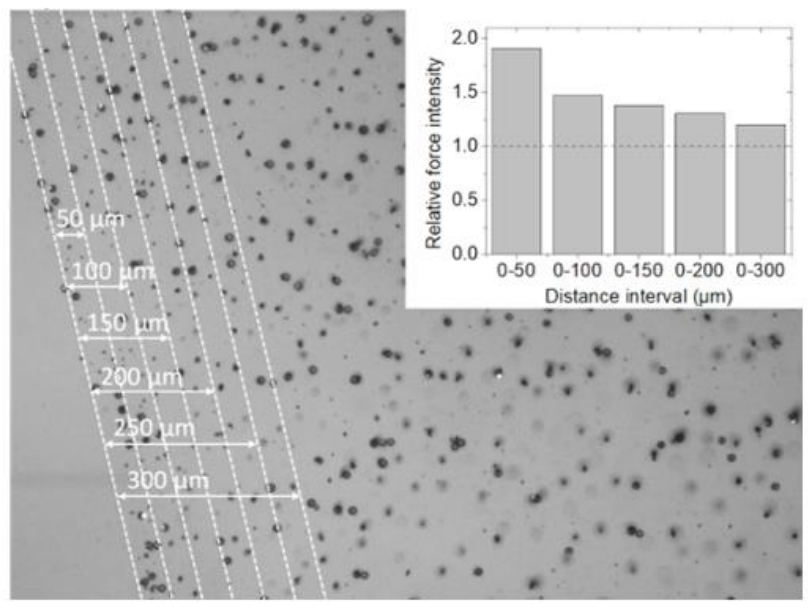

b)

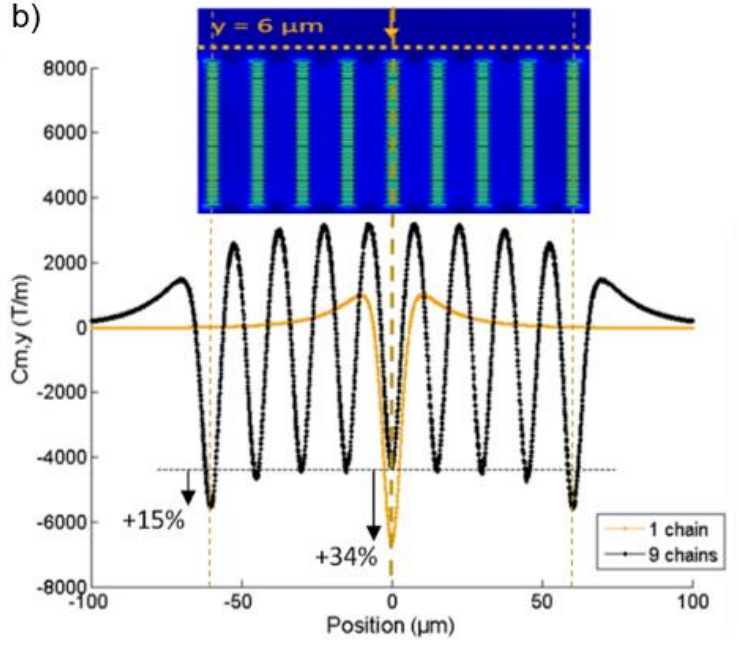

Figure 5. (a) Calculation of the magnetic coefficient generated above a network of nine chains and comparison with a single chain. (b) Relative force intensity according to the position in the microchannel. The relative force intensity refers to the ratio of the measured force in one position in the channel to the average force in the whole channel.

We also compared the forces generated by the same trap in the presence or absence of the external magnet. Although these measurements were performed with different trapped beads, whose diameters varied from 8 to $20 \mu \mathrm{m}$ (see Figure S7 for SEM image of the SPMBs), we observed, on 192 traps, that for 70\% of them the magnetic forces were greater or equal in the presence of the magnet.

\subsection{Implementation of the Micro-Traps in Microsystems for the Manipulation of Magnetic Entities}

In order to evaluate the sorting potential of micro-magnets for biomedical applications, we first evaluated the trapping efficiency of SPMB models and then demonstrated the trapping of magnetically functionalized cells. The magnetophoretic trapping efficiency of the micro-magnets integrated into the microfluidic system was determined on a sample of 6500 SPMBs at various flow rates (Figure $6 a$ ). At $0.5 \mathrm{~mL} / \mathrm{h}, 85 \%$ and $100 \%$ of injected beads are trapped in the absence and in the presence of the milli-magnet, respectively. 
At this flow rate, the trapping throughput is about 400 beads $/ \mathrm{min}$. The trapping area, being $7.5 \mathrm{~mm}^{2}$, has a trapping density of 750 beads $/ \mathrm{mm}^{2}$ when all beads are trapped. At $1 \mathrm{~mL} / \mathrm{h}$, the improvement from adding milli-magnets is even more pronounced. The trapping efficiency reaches $75 \%$ with the combination of micro- and milli-magnets, whereas it is $50 \%$ with the micro-traps alone. Indeed, from COMSOL calculations of the magnetic field value along the 100- $\mu \mathrm{m}$ channel height (Figure 3b), the magnetization of the flowing SPMBs is significantly increased throughout the channel in the presence of the milli-magnet. Besides, as shown previously by COMSOL simulations, at a distance greater than $50 \mu \mathrm{m}$ above micro-traps, the magnetic field gradient generated by the milli-magnet predominates (Figure $3 \mathrm{~b}$ ) and allows flowing targets located in the upper part of the channel to be dragged down. The strong and localized magnetic field gradients generated by the micro-magnets then efficiently retain the trapped targets. It is worth mentioning that the milli-magnet alone leads to significantly less trapping efficiency, as compared with the micro-magnets. Indeed, the generated gradients at the channel surface are more than three orders of magnitude lower than those generated by the micro-magnets $\left(30 \mathrm{~T} / \mathrm{m}\right.$ vs. $\left.10^{5} \mathrm{~T} / \mathrm{m}\right)$, which results in a trapping efficiency of only $48 \%$ at $0.5 \mathrm{~mL} / \mathrm{h}$ with the milli-magnet alone. It is therefore the combination of the two types of magnet that allows higher magnetic forces to be generated, which explains the higher trapping efficiencies. Other works rely on the association of passive and magnetic functions to attract the targets towards the magnets. For example, Chung et al. implemented herringbone structures on the top of the channel to deflect magnetic objects to the surface of the magnetic traps by chaotic mixing [33]. Beyond $2.5 \mathrm{~mL} / \mathrm{h}$, the benefit of the external milli-magnet becomes negligible in comparison with the drag force. Thus, circulating micro-beads which are far from the traps are no longer attracted to the latter with the external magnet. The trapping performances are therefore identical for both configurations.
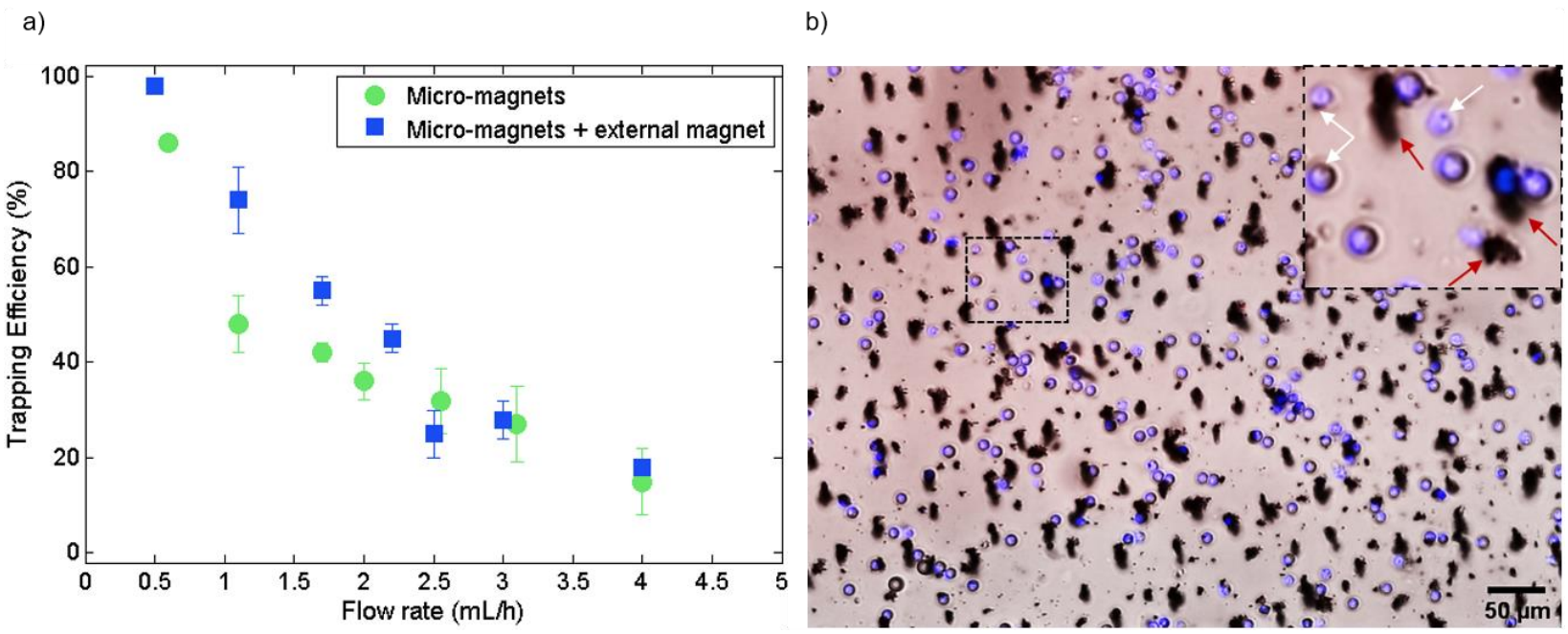

Figure 6. (a) Magnetic trapping efficiency of 12- $\mu \mathrm{m}$ SPMBs on the micro-magnets as a function of the flow rate, with or without the external magnet. (b) Composite microscope image showing trapped magnetically labeled WBCs (blue) in the microfluidic channel at $200 \mu \mathrm{L} / \mathrm{h}$. All white blood cells are trapped on magnetic traps. What appears to be large traps are instead agglomerates of magnetic nanoparticles (red arrows in the inset). The inset highlights the trapping of white blood cells on less visible magnetic traps (white arrows).

Finally, the biocompatibility and operation of the trapping device were established with biological samples by successful WBC depletion. Specific capture of magnetically labeled WBCs on the micro-traps is illustrated in Figure 6b. Spiked PC-3 cancer cells were recovered at the output; therefore, the device could be easily implemented for cell sorting based on their magnetic functionalities. 


\section{Conclusions}

We demonstrated an original approach to integrate large density arrays of permanent micro-magnets into a microfluidic system. This approach, based on hard magnetic powderpolymer composites, led to autonomous and compact systems that were successfully implemented for the manipulation of SPMBs and the sorting of magnetically labeled cells. The integrated micro-magnets can generate magnetic forces of several $\mathrm{nN}$, which is comparable to the magnetic force generated by micro-scale magnetic sources obtained by more conventional and complex microfabrication methods. We identified high magnetic field gradients generated by the integrated micro-magnets of up to $10^{5} \mathrm{~T} / \mathrm{m}$ at their surface. We used an external milli-magnet to add a background field throughout the channel and quantified its effect on magnetic forces and trapping efficiency. This was conducted using three approaches: numerical simulations, colloidal probe AFM measurements, and fluidic experiments. In particular, we demonstrated that this additional field led to an increase in the trapping efficiency for flow rates of between 0.5 and $2.5 \mathrm{~mL} / \mathrm{h}$. At $0.5 \mathrm{~mL} / \mathrm{h}$, the trapping efficiency rose from $85 \%$ to $100 \%$. Moreover, cell isolation was performed through the depletion of magnetically labelled white blood cells. These cost-effective functional materials integrating micro-magnet arrays open the way to a broad range of magnetophoretic applications, especially in the biomedical field: from the detection of biological molecules via the manipulation of functionalized magnetic microbeads, to immunomagnetic separation requiring the manipulation of thousands of target objects in a blood sample.

Supplementary Materials: The following are available online at https://www.mdpi.com/article/ 10.3390/cells10071734/s1, Figure S1: SEM image of NdFeB particles, Figure S2: Approach/retract curve from colloidal probe AFM measurement, Figure S3: Picture of the experimental set-up, Figure S4: Magnetization curve of the superparamagnetic beads, Figure S5: Magnetization curve of the $\mathrm{NdFeB}$ particles, Equation and numerical simulation of the magnetic field gradient, Figure S6: Numerical simulations of a network of increasing chain number, Figure S7: SEM image of superparamagnetic beads.

Author Contributions: Conceptualization, L.D., S.M., D.L.R. and A.-L.D.; methodology, L.D., M.C.A., S.M., C.A., E.L., D.L.R. and A.-L.D.; validation, L.D., D.L.R. and A.-L.D.; formal analysis, L.D., M.-C.A. and D.L.R.; investigation, L.D., J.H., M.-C.A., D.L.R. and A.-L.D.; resources, D.B., L.P. and J.G.; writing-original draft preparation, L.D., D.L.R. and A.-L.D.; writing-review and editing, L.D., J.H., S.M., M.-C.A., E.L., D.L.R. and A.-L.D.; visualization, L.D., M.-C.A., A.-L.D. and D.L.R.; supervision, D.L.R. and A.-L.D. All authors have read and agreed to the published version of the manuscript.

Funding: This research was funded by PACK AMBITION RECHERCHE AuRA, grant LUTON number 1701103701-40890.

Institutional Review Board Statement: Not applicable.

Informed Consent Statement: Not applicable.

Data Availability Statement: Not applicable.

Acknowledgments: The authors acknowledge support staff from Nanolyon technological platform, MATEIS lab for X-ray tomography measurements, and ILM-Tech for AFM and SQUID facilities. All the staff of the CIRCAN team from the Hospices Civils de Lyon is also gratefully acknowledged for their help on biological sample preparation.

Conflicts of Interest: The authors declare no conflict of interest.

\section{References}

1. Alnaimat, F.; Karam, S.; Mathew, B.; Mathew, B. Magnetophoresis and Microfluidics: A Great Union. IEEE Nanotechnol. Mag. 2020, 14, 24-41. [CrossRef]

2. Deman, A.-L.; Le Roy, D. Magnetophoresis in Bio-Devices. In Engineering of Micro/Nano Biosystems; Springer: Singapore, 2020; pp. 309-361. 
3. Bejhed, R.S.; Tian, B.; Eriksson, K.; Brucas, R.; Oscarsson, S.; Strömberg, M.; Svedlindh, P.; Gunnarsson, K. Magnetophoretic Transport Line System for Rapid On-Chip Attomole Protein Detection. Langmuir 2015, 31, 10296-10302. [CrossRef]

4. Zirath, H.; Schnetz, G.; Glatz, A.; Spittler, A.; Redl, H.; Peham, J.R. Bedside Immune Monitoring: An Automated Immunoassay Platform for Quantification of Blood Biomarkers in Patient Serum within 20 Minutes. Anal. Chem. 2017, 89, 4817-4823. [CrossRef]

5. Gao, Y.; Huo, W.; Zhang, L.; Lian, J.; Tao, W.; Song, C.; Tang, J.; Shi, S.; Gao, Y. Multiplex measurement of twelve tumor markers using a GMR multi-biomarker immunoassay biosensor. Biosens. Bioelectron. 2019, 123, 204-210. [CrossRef]

6. Hung, P.Y.; Jiang, P.S.; Lee, E.F.; Fan, S.K.; Lu, Y.W. Genomic DNA extraction from whole blood using a digital microfluidic (DMF) platform with magnetic beads. Microsyst. Technol. 2015, 23, 313-320. [CrossRef]

7. Dias, T.M.; Cardoso, F.A.; Martins, S.A.M.; Martins, V.C.; Cardoso, S.; Gaspar, J.F.; Monteiro, G.; Freitas, P.P. Implementing a strategy for on-chip detection of cell-free DNA fragments using GMR sensors: A translational application in cancer diagnostics using ALU elements. Anal. Methods 2016, 8, 119-128. [CrossRef]

8. Garbarino, F.; Minero, G.A.S.; Rizzi, G.; Fock, J.; Hansen, M.F. Integration of rolling circle amplification and optomagnetic detection on a polymer chip. Biosens. Bioelectron. 2019, 142, 111485. [CrossRef] [PubMed]

9. Poudineh, M.; Sargent, E.H.; Kelley, S.O. Amplified Micromagnetic Field Gradients Enable High-Resolution Profiling of Rare Cell Subpopulations. ACS Appl. Mater. Interfaces 2017, 9, 25683-25690. [CrossRef]

10. Aldridge, P.M.; Mukhopadhyay, M.; Ahmed, S.U.; Zhou, W.; Christinck, E.; Makonnen, R.; Sargent, E.H.; Kelley, S.O. Prismatic Deflection of Live Tumor Cells and Cell Clusters. ACS Nano 2018, 12, 12692-12700. [CrossRef] [PubMed]

11. Mishra, A.; Dubash, T.D.; Edd, J.F.; Jewett, M.K.; Garre, S.G.; Karabacak, N.M.; Rabe, D.C.; Mutlu, B.R.; Walsh, J.R.; Kapur, R.; et al. Ultrahigh-throughput magnetic sorting of large blood volumes for epitope-agnostic isolation of circulating tumor cells. Proc. Natl. Acad. Sci. USA 2020, 117, 16839-16847. [CrossRef]

12. Cao, Q.; Fan, Q.; Chen, Q.; Liu, C.; Han, X.; Li, L. Recent advances in manipulation of micro- and nano-objects with magnetic fields at small scales. Mater. Horiz. 2020, 7, 638-666. [CrossRef]

13. Fratzl, M.; Delshadi, S.; Devillers, T.; Bruckert, F.; Cugat, O.; Dempsey, N.M.; Blaire, G. Magnetophoretic induced convective capture of highly diffusive superparamagnetic nanoparticles. Soft Matter 2018, 14, 2671-2681. [CrossRef] [PubMed]

14. Huang, Y.Y.; Chen, P.; Wu, C.H.; Hoshino, K.; Sokolov, K.; Lane, N.; Liu, H.; Huebschman, M.; Frenkel, E.; Zhang, J.X.J. Screening and Molecular Analysis of Single Circulating Tumor Cells Using Micromagnet Array. Sci. Rep. 2015, 5, 16047. [CrossRef] [PubMed]

15. Walther, A.; Marcoux, C.; Desloges, B.; Grechishkin, R.; Givord, D.; Dempsey, N.M. Micro-patterning of NdFeB and SmCo magnet films for integration into micro-electro-mechanical-systems. J. Magn. Magn. Mater. 2009, 321, 590-594. [CrossRef]

16. Owen, D.; Ballard, M.; Alexeev, A.; Hesketh, P.J. Rapid microfluidic mixing via rotating magnetic microbeads. Sens. Actuators A Phys. 2016, 251, 84-91. [CrossRef]

17. Le Roy, D.; Shaw, G.; Haettel, R.; Hasselbach, K.; Dumas-Bouchiat, F.; Givord, D.; Dempsey, N.M. Fabrication and characterization of polymer membranes with integrated arrays of high performance micro-magnets. Mater. Today Commun. 2016, 6, 50-55. [CrossRef]

18. Dempsey, N.M.; Le Roy, D.; Marelli-Mathevon, H.; Shaw, G.; Dias, A.; Kramer, R.B.G.; Viet Cuong, L.; Kustov, M.; Zanini, L.F.; Villard, C.; et al. Micro-magnetic imprinting of high field gradient magnetic flux sources. Appl. Phys. Lett. $2014,104,262401$. [CrossRef]

19. Mekkaoui, S.; Descamps, L.; Audry, M.C.; Deman, A.L.; Le Roy, D. Nanonewton Magnetophoretic Microtrap Array for Microsystems. Langmuir 2020, 36, 14546-14553. [CrossRef]

20. Mekkaoui, S.; Le Roy, D.; Audry, M.C.; Lachambre, J.; Dupuis, V.; Desgouttes, J.; Deman, A.L. Arrays of high aspect ratio magnetic microstructures for large trapping throughput in lab-on-chip systems. Microfluid. Nanofluidics 2018, 22, 119. [CrossRef]

21. Le Roy, D.; Dhungana, D.; Ourry, L.; Faivre, M.; Ferrigno, R.; Tamion, A.; Dupuis, V.; Salles, V.; Deman, A.L. Anisotropic ferromagnetic polymer: A first step for their implementation in microfluidic systems. AIP Adv. 2016, 6, 056604. [CrossRef]

22. Marchi, S.; Casu, A.; Bertora, F.; Athanassiou, A.; Fragouli, D. Highly Magneto-Responsive Elastomeric Films Created by a Two-Step Fabrication Process. ACS Appl. Mater. Interfaces 2015, 7, 19112-19118. [CrossRef] [PubMed]

23. Malic, L.; Zhang, X.; Brassard, D.; Clime, L.; Daoud, J.; Luebbert, C.; Barrere, V.; Boutin, A.; Bidawid, S.; Farber, J.; et al. Polymer-based microfluidic chip for rapid and efficient immunomagnetic capture and release of Listeria monocytogenes. Lab Chip 2015, 15, 3994-4007. [CrossRef] [PubMed]

24. Zhou, R.; Yang, Q.; Bai, F.; Werner, J.A.; Shi, H.; Ma, Y.; Wang, C. Fabrication and integration of microscale permanent magnets for particle separation in microfluidics. Microfluid. Nanofluidics 2016, 20, 110. [CrossRef]

25. Royet, D.; Hériveaux, Y.; Marchalot, J.; Scorretti, R.; Dias, A.; Dempsey, N.M.; Bonfim, M.; Simonet, P.; Frénéa-Robin, M. Using injection molding and reversible bonding for easy fabrication of magnetic cell trapping and sorting devices. J. Magn. Magn. Mater. 2017, 427, 306-313. [CrossRef]

26. Lin, S.; Zhi, X.; Chen, D.; Xia, F.; Shen, Y.; Niu, J.; Huang, S.; Song, J.; Miao, J.; Cui, D.; et al. A flyover style microfluidic chip for highly purified magnetic cell separation. Biosens. Bioelectron. 2019, 129, 175-181. [CrossRef] [PubMed]

27. Hu, X.; Goudu, S.R.; Torati, S.R.; Lim, B.; Kim, K.; Kim, C. An on-chip micromagnet frictionometer based on magnetically driven colloids for nano-bio interfaces. Lab Chip 2016, 16, 3485-3492. [CrossRef] [PubMed] 
28. Bongaerts, M.; Aizel, K.; Secret, E.; Jan, A.; Nahar, T.; Raudzus, F.; Neumann, S.; Telling, N.; Heumann, R.; Siaugue, J.-M.; et al. Parallelized Manipulation of Adherent Living Cells by Magnetic Nanoparticles-Mediated Forces. Int. J. Mol. Sci. 2020, $21,6560$. [CrossRef]

29. Ponomareva, S.; Dias, A.; Royer, B.; Marelli, H.; Motte, J.F.; Givord, D.; Dumas-Bouchiat, F.; Dempsey, N.M.; Marchi, F. A quantitative study of magnetic interactions between a micro-magnet array and individual magnetic micro-particles by scanning particle force microscopy. J. Micromech. Microeng. 2019, 29, 015010. [CrossRef]

30. Furlani, E.P. Analysis of particle transport in a magnetophoretic microsystem. J. Appl. Phys. 2006, 99, 024912. [CrossRef]

31. Gerber, R.; Takayasu, M.; Friedlaender, F. Generalization of HGMS theory: The capture of ultra-fine particles. IEEE Trans. Magn. 1983, 19, 2115-2117. [CrossRef]

32. Mirowski, E.; Moreland, J.; Russek, S.E.; Donahue, M.J. Integrated microfluidic isolation platform for magnetic particle manipulation in biological systems. Appl. Phys. Lett. 2004, 84, 1786-1788. [CrossRef]

33. Chung, J.; Issadore, D.; Ullal, A.; Lee, K.; Weissleder, R.; Lee, H. Rare cell isolation and profiling on a hybrid magnetic/size-sorting chip. Biomicrofluidics 2013, 7, 054107. [CrossRef] [PubMed] 